\title{
INTERNACIONALIZAÇÃO E SUSTENTABILIDADE EMPRESARIAL NO BRASIL
}

\author{
Ítalo Carlos Soares do Nascimento ${ }^{1}$, Andressa Ruth Sousa Santos, Adriano Fleck de Paula Pessoa, Daniel Barboza \\ Guimarães, Sílvia Maria Dias Pedro Rebouças \\ Universidade Federal do Ceará - UFC, Fortaleza, CE (Brasil)
}

\section{DETALHES DO ARTIGO}

\section{Histórico do Artigo:}

Recebido: 06 de Janeiro de 2020

Aceito: 30 de Junho de 2020

Disponível online: 01 de Agosto de 2020

Sistema de revisão "Double blind review"

Editor Científico

Ilan Avrichir

\section{Palavras-chaves:}

Internacionalização

Sustentabilidade Empresarial

Teoria dos Stakeholders

\begin{abstract}
RESUMO
Objetivo: Investigar a relação entre a internacionalização e a sustentabilidade empresarial em companhias listadas na Brasil, Bolsa, Balcão (B3 S.A.).

Método: A amostra reúne 95 das 100 empresas listadas na B3 S.A. com maior valor de mercado, de acordo com dados extraídos do Economática ${ }^{\circledR}$. Os dados referemse ao exercício social de 2017, sendo coletados em novembro de 2018, utilizandose o teste de diferenças entre médias e a regressão logística para a análise quantitativa.

Principais resultados: $O$ resultado do teste de diferenças entre médias revelou que apenas as empresas internacionalizadas por meio da presença física em outros países apresentaram diferenças estatisticamente significantes no tocante à sustentabilidade empresarial, o que também foi ratificado com a aplicação da regressão logística, constatando-se que a internacionalização através do capital social e das receitas não demonstrou relação com a sustentabilidade empresarial. Dessa forma, rejeita-se a hipótese de pesquisa de que há uma relação positiva entre a internacionalização e a sustentabilidade empresarial no Brasil.

Relevância/originalidade: A sinergia entre os dois constructos - Internacionalização e Sustentabilidade Empresarial -, evidenciada em pesquisas estrangeiras, tem demonstrado a relevância desse debate. No âmbito nacional, por sua vez, não foram encontradas evidências empíricas que tratassem da relação entre as variáveis estudadas.

Contribuições teóricas/metodológicas: O estudo da internacionalização e da sustentabilidade empresarial proporciona reflexões sobre a busca pelo valor a longo prazo, permitindo assim visualizar a Teoria dos Stakeholders, a qual sustenta que as empresas, para garantir valor a longo prazo, necessitam conciliar os interesses dos diversos stakeholders envolvidos.
\end{abstract}

\section{INTRODUÇÃO}

A internacionalização leva as empresas a responder as demandas das partes interessadas através do aumento de práticas de responsabilidade social corporativa (Attig, Boubakri, Ghoul \& Guedhami, 2016). Além disso, aumenta o risco gerencial, uma vez que as empresas multinacionais dependem mais das habilidades específicas e experiências dos executivos (Kang, 2013). Desta forma, os gerentes de empresas internacionais alocam maiores recursos para atender os interesses dos stakeholders (Kacperczyk, 2009). Neste contexto, estudos como o de Kang (2013) mostram que há uma maior preocupação de organizações internacionais em relação às práticas de responsabilidade social.
Outros demonstram que, cada vez mais, investidores preferem empresas que possuam práticas de responsabilidade social (Scholtens \& Sievänen 2013; Sievänen, Rita \& Scholten, 2013).

Além disso, organizações multinacionais, por trabalharem em diferentes países e lidarem com diversos sistemas legais e interesses de indivíduos, trabalham a questão da sustentabilidade de forma diferenciada. Diferentemente de empresas que possuem apenas um mesmo tipo de exigência de uma região, firmas internacionais possuem demandas mais complexas e interações que possam influenciar suas práticas. Desta forma, alguns estudiosos trazem evidências empíricas de que a internacionalização é positiva e significativamente relacionada às práticas de responsabilidade social

\footnotetext{
${ }^{1}$ Contato do autor e-mail: italocarlos25@gmail.com
} 
corporativa (Kolk \& Van Tulder, 2010; Attig, Boubakri, Ghoul \& Guedhami, 2016; Chakrabarty \& Wang, 2012; Hörisch, Burritt, Christ \& Schaltegger, 2017; Symeou, Zyglidopoulos \& Williamson, 2017).

Com o interesse pela questão, grandes bolsas de valores do mundo, como as de Nova lorque, Londres e São Paulo criaram índices baseados nessas três dimensões - econômica, social e ambiental: Dow Jones Sustainability Index (DJSI), FTSE4Good e o Índice de Sustentabilidade Empresarial (ISE), respectivamente. A importância dessa questão, fora a preocupação ambiental e social, também passa pelo próprio valor da organização que está cada vez mais relacionado com o interesse de toda a comunidade envolvida com as atividades da empresa, conforme estabelecido na Teoria dos Stakeholders (Freeman, 1984; Jensen, 2001). Desta forma, a sinergia entre os dois constructos Internacionalização e Sustentabilidade Empresarial , evidenciada em pesquisas estrangeiras, tem demonstrado a relevância desse debate. Além disso, o estudo tem como base os preceitos da Teoria dos Stakeholders, a qual afirma que a organização deve se preocupar não só com o shareholders, mas também com os diferentes grupos envolvidos e seus interesses específicos (Donaldson \& Preston, 1995).

Uma vez que as ações de responsabilidade social vão além do interesse da organização e que são influenciadas pela internacionalização desta (McWilliams \& Siegel, 2001), seguindo as evidências teóricas apresentadas, emerge a seguinte questãoproblema: Qual a relação entre a internacionalização e a sustentabilidade empresarial em companhias listadas na B3 S.A.? Destarte, constitui objetivo geral deste estudo, investigar a relação entre a internacionalização e a sustentabilidade empresarial em companhias listadas na B3 S.A. Para o alcance do objetivo, foi realizada uma pesquisa descritiva, quantitativa e documental, aplicando-se o teste de diferenças entre médias e a regressão logística, com base nos dados do exercício social de 2017, extraídos da base Economática ${ }^{\circledR}$ e do Formulário de Referência das empresas da amostra listadas na B3 S.A., além de informações contidas em Índices e Rankings de Sustentabilidade.

Destarte, esta pesquisa justifica-se por abordar temática atual, relevante e pouco explorada em âmbito nacional, tanto para o ambiente acadêmico como para o ambiente empresarial. Do ponto de vista acadêmico, espera-se contribuir para o aprofundamento e avanço da discussão dessas questões, trazendo novas constatações e procurando preencher lacunas ainda existentes, especialmente no contexto brasileiro, por se tratar de um país emergente e considerando-se que as pesquisas já realizadas se deram em países desenvolvidos, diferenciando-se, portanto, dos estudos anteriores. Além disso, o estudo apresenta uma abordagem metodológica multidimensional, que permite a análise da estratégia de internacionalização sobre múltiplas perspectivas para a investigação proposta. Para o mercado, é notória a crescente discussão sobre a sustentabilidade empresarial e quais são os fatores que corroboram para essas práticas no ambiente corporativo. Entender a internacionalização como parte desse processo é fundamental para as empresas que buscam crescimento, competitividade e continuidade, tendo em vista o engajamento com os seus diversos stakeholders. Destarte, com o estudo pretende-se avaliar se a internacionalização é um fator que contribui para o desenvolvimento de boas práticas sustentáveis das organizações brasileiras.

O artigo inicia-se pela discussão da internacionalização de empresas e da sustentabilidade empresarial, buscando-se enfatizar os principais aspectos presentes nos dois constructos. A discussão teórica e as lacunas deixadas pelos estudos empíricos anteriores embasam a hipótese levantada. Em seguida, apresenta-se a metodologia, a base de dados e o modelo adotado, apresentando-se na sequência a análise e discussão dos resultados da relação entre internacionalização e sustentabilidade empresarial. Por fim, são sumarizadas as contribuições do estudo e os desafios para pesquisas futuras.

\section{FUNDAMENTAÇÃO TEÓRICA}

Esta seção discute os temas internacionalização de empresas e sustentabilidade empresarial. Além disso, são destacados os resultados de alguns estudos empíricos anteriores que verificaram a associação entre os constructos.

\section{Internacionalização de Empresas}

Após a abertura da economia no início dos anos 1990, as empresas brasileiras não apenas passaram a sofrer o impacto do aumento da concorrência externa, como também perceberam a relevância de 
ampliar seu mercado via implementação de estratégias de internacionalização, utilizando vantagens competitivas existentes ou passíveis de ser obtidas (Machado-da-Silva \& Fernandes, 1999; Platchek, Floriani \& Borini, 2012). A redução das barreiras comerciais e os incentivos do governo na época, bem como a modernização dos meios de comunicação e a melhoria dos meios de transporte abriram caminhos para a internacionalização dessas empresas, ao ampliar o leque de opções de localização de linhas de produção, assim como para o enfrentamento de novos desafios (Denberg \& Gomes, 2011). Destaca-se, assim, que parcerias estrangeiras, investimentos estrangeiros e agrupamentos internacionais representam caminhos exequíveis para facilitar o intercâmbio do conhecimento e das estratégias empresariais (Bouças \& Gomes, 2010).

A internacionalização pode impactar de forma significativa as estruturas das empresas, devido às exigências competitivas do mercado internacional, para além daquelas cumpridas apenas no país de origem, demandando exame multidimensional, já que engloba, além do envolvimento internacional, a adaptação estratégica de recursos e da estrutura, evidenciando-se essa visão como a mais apropriada à análise da conveniência e oportunidade de internacionalização (Floriani \& Fleury, 2012; Santos, Vasconcelos \& De Luca, 2015).

São inúmeras as motivações para a internacionalização, abrangendo aspectos como, por exemplo, a expansão dos mercados, a redução da dependência do mercado interno, a proteção contra ciclos econômicos desfavoráveis, a busca de conhecimento e o acesso a recursos naturais ou menores custos dos fatores (Ribeiro, Serra \& Bertolini, 2016). Nesse contexto, observa-se que as empresas internacionalizadas procuram se beneficiar das vantagens decorrentes da criação de subsidiárias em outros países, impulsionadas por diferentes estratégias, envolvendo incertezas, riscos, complexidade e custos, que podem ser compensados pelos benefícios resultantes da expansão, destacando-se o crescimento da firma, a conquista de novos mercados, a economia de escala e o fortalecimento da posição competitiva (Silva, Coronel, Freitas \& Silva, 2015).

Apesar dos inúmeros benefícios da internacionalização, na maioria das empresas as operações internacionais iniciam-se bem mais tarde que as operações no mercado interno. Destarte, por um tempo considerável correspondem a um pequeno percentual das receitas e dos custos da empresa. Enquanto são embrionárias, tais operações costumam ser tratadas como marginais; ou seja, pode não haver uma explicitação ou um controle dos custos correspondentes (Platchek, Floriani \& Borini, 2012).

Vários estudos procuram mensurar e identificar o grau de internacionalização de empresas. Pioneiro nesse tipo de abordagem, Sullivan (1994) criou uma medida de internacionalização de empresas, denominada Degree of Internationalization, por meio da análise de cinco elementos - proporção de vendas no exterior em relação ao total, proporção de ativos estrangeiros, proporção de subsidiárias no exterior, experiência internacional de membros da gerência e da diretoria e dispersão psíquica de operações internacionais.

Destaca-se também o estudo de Vermeulen e Barkema (2002), o qual constata que o ritmo e os passos da expansão internacional das empresas resultam em diferentes tipos de performance. Para isso, os autores analisaram três dimensões - período, extensão geográfica e regularidade do processo de internacionalização - concluindo que as empresas que se internacionalizaram com um padrão regular, beneficiaram-se mais do que outras que realizaram esse processo de forma irregular. Apesar disso, segundo Cuervo-Cazurra, Maloney e Manrakhan (2007), dependendo do país em que a empresa busca esse processo, três fatores podem vir a comprometer seu desempenho, além da falta de regularidade do padrão de internacionalização: discriminação de consumidores e governo contra companhias estrangeiras; perda de vantagem, quando recursos deixam de ser benéficos, por serem transferidos para um novo mercado internacional; e falta de recursos complementares no novo mercado.

Em estudo mais recente, evidencia-se que a análise sobre internacionalização de empresas em países em desenvolvimento deve ser diferente daquela feita em economias desenvolvidas, pois naqueles há diferentes padrões, que não são corretamente contemplados na maioria dos estudos sobre a temática (Cuervo-Cazurra, 2012).

Desse modo, há divergências entre as formas de se estudar o grau de internacionalização das empresas, em especial as brasileiras. Dentre as métricas mais comuns de internacionalização 
utilizadas em estudos recentes com empresas listadas na bolsa de valores brasileira (Mapurunga, Ponte \& Oliveira, 2015; Santos, Almeida \& Bezerra, 2013; Santos, Vasconcelos \& De Luca, 2015; Souza, Murcia \& Marcon, 2011), destacam-se as seguintes: emissão de American Depositary Receipt (ADR); internacionalização do capital social - parcela de estrangeiros acionistas; internacionalização das receitas - provenientes das receitas estrangeiras (exportações); e internacionalização de mercados número de países onde atua a empresa (instalação de subsidiárias). Para atender o objetivo do presente estudo, foram utilizadas as três últimas.

\section{Sustentabilidade Empresarial}

A partir da década de 1970, a questão da sustentabilidade se tornou relevante e passou a compor a pauta do planejamento de governos, empresas, organizações não governamentais (ONG) e organismos internacionais (Ackerman, 1975; Dyllick \& Hockerts, 2002; Ackerman, 1975; McDonald \& Puxty, 1979). Apesar de complexa conceituação, a sustentabilidade ficou conhecida como um conjunto de práticas que compreendem o equilíbrio entre o impacto econômico, social e ambiental, o tripé de Elkington (2001). Independentemente de até hoje muitas dessas práticas sustentáveis ainda não serem exigidas por lei, a Teoria dos Stakeholders fundamenta que organizações devem adotar práticas que atendam não apenas o interesse dos shareholders, mas de toda a sociedade que pode ser impactada por ações irresponsáveis (Donaldson \& Preston, 1995; Irene \& Robin, 2002). Ou seja, os gestores devem tomar suas decisões respeitando o bem-estar dos stakeholders, pois estes não são apenas meios para um fim empresarial (Freeman \& McVea, 2001).

Neste contexto, observa-se que informações sobre o comportamento socialmente responsável é um tema de crescente interesse dos stakeholders, os quais consideram uma empresa socialmente responsável quando esta se envolve em questões sociais, bem como desenvolve capacidade de resposta às demandas dos interessados, mostrandose responsável para com a sociedade de forma voluntária (Kim \& Kim; Soschinski, Brandt \& Klann, 2019).

A Teoria dos Stakeholders, de acordo com Freeman (1984), explica a relação entre as organizações e os diferentes interesses dos usuários das informações. Tais usuários, por sua vez, podem ser definidos por diversos grupos, incluindo clientes, fornecedores, empregados, acionistas, investidores, comunidade e todos aqueles que possam afetar ou ser afetados pelas decisões da empresa, sendo denominados de stakeholders.

Ainda sob a ótica da Teoria dos Stakeholders, a organização é um sistema de stakeholders, e sua continuidade está atrelada à capacidade que tem de cumprir sua finalidade econômica e social, gerando valor ou riqueza (Clarkson, 1995). Assim, a teoria se interessa pela natureza das relações entre a organização e seus stakeholders em termos de processos e resultados e ao processo de tomada de decisão dos gestores, permitindo, portanto, explicar a razão pela qual as companhias devem adotar condutas socialmente responsáveis (Donaldson \& Preston, 1995).

Como forma de mensurar a responsabilidade das organizações em respeito à questão sustentável, uma série de índices, rankings e recomendações foram criadas por diversas organizações, ONGs e bolsas de valores. Dentre esses índices, um dos primeiros de grande destaque foi o Dow Jones Sustainability Index (DJSI). Lançado em 1999, é um indicador da bolsa de valores de Nova Iorque que alia desempenho econômico, social e ambiental e passou a indicar quais são a principais organizações sustentáveis dos Estados Unidos (Knoepfel, 2001).

Após dois anos da criação do DJSI, surgiu o FTSE4Good que derivou do índice Financial Times Stock Exchange (FTSE) da Grã-Bretanha-do grupo do Jornal Financial Times - e passou a incorporar dados de países de várias regiões, como Europa, América e Ásia. Seguindo essa tendência, em 2004, foi criado o primeiro índice de sustentabilidade de um país emergente, o Socially Responsible Index (SRI) da bolsa de valores de Johanesburgo, da África do Sul (Guay, Doh \& Sinclair, 2004). No ano seguinte, em 2005, surge no Brasil o Índice de Sustentabilidade Empresarial (ISE) da Bolsa de Valores, Mercadorias e Futuros (BM\&FBOVESPA), cuja metodologia e produção foram desenvolvidas pela Fundação Getúlio Vargas (FGV). Para participação no ISE é necessário estar entre as 200 ações com maior liquidez da bolsa de valores de São Paulo, além de responder a um questionário-base sobre questões sustentáveis realizadas pela organização. Após isso, até 40 empresas vão compor o índice no primeiro dia 
do ano seguinte, sendo assim, atualizado anualmente (Machado, Machado \& Corrar, 2009).

Apesar do ISE, a BM\&FBOVESPA - denominada B3 S.A. Brasil, Bolsa, Balcão, desde junho de 2017 juntamente com o Banco Nacional de Desenvolvimento Econômico e Social (BNDES) decidiram criar um novo índice sustentável, o Índice Carbono Eficiente (ICO2), o qual é composto pelas ações das companhias participantes do índice IBrX-50 que aceitaram participar desta iniciativa, adotando práticas transparentes com relação a suas emissões de gases efeito estufa. Cabe ressaltar que a B3 S.A. é a atual bolsa de valores brasileira, considerada como uma das principais empresas de infraestrutura de mercado financeiro no mundo, com atuação em ambiente de bolsa e de balcão (B3 S.A., 2017).

Além dos índices, pode-se destacar também o Guia Exame de Sustentabilidade que publica o ranking das empresas que se utilizam das mais avançadas práticas sustentáveis no Brasil (Furlan, 2013) para a análise de sustentabilidade organizacional. A Exame também publica o ranking das Maiores e Melhores empresas brasileiras. Apesar desse índice ser mais orientado para a dimensão econômica, ele se utiliza de diversos critérios, dentre os quais estão os sustentáveis. Por último, destaca-se também o ranking das 150 Melhores Empresas Para Trabalhar da Revista Você S.A., que ressalta a questão social, uma vez que considera principalmente, dentre os seus critérios, itens e vantagens do ponto de vista do trabalhador.

\section{Internacionalização e Sustentabilidade Empresarial}

Advoga-se que o processo de internacionalização, pelo qual as empresas passam, impacta as suas estruturas devido às exigências competitivas do mercado internacional. Ou seja, ao expandir suas fronteiras, as organizações têm de se adequar a novas demandas legais, sociais e culturais, uma vez que engloba, além do envolvimento internacional, a adaptação estratégica de recursos e da estrutura, evidenciando-se essa visão como a mais apropriada à análise da conveniência e oportunidade de internacionalização (Floriani \& Fleury, 2012; Santos, Vasconcelos \& De Luca, 2015; Albuquerque Filho, Freire, De Luca \& Vasconcelos, 2020).

No entanto, há muitas vantagens para a internacionalização, abrangendo aspectos como, por exemplo, a expansão dos mercados, a redução da dependência do mercado interno, a proteção contra ciclos econômicos desfavoráveis, a busca de conhecimento e o acesso a recursos naturais ou menores custos dos fatores (Ribeiro, Serra \& Bertolini, 2016). Dessa forma, observa-se que as empresas internacionalizadas procuram se beneficiar das vantagens decorrentes da criação de subsidiárias em outros países, impulsionadas por diferentes estratégias, envolvendo incertezas, riscos, complexidade e custos, que podem ser compensados pelos benefícios resultantes da expansão, destacando-se o crescimento da firma, a conquista de novos mercados, a economia de escala e o fortalecimento da posição competitiva (Silva, Coronel, Freitas \& Silva, 2015).

Em contrapartida à internacionalização e à luz da Teoria dos Stakeholders, as organizações para serem bem-sucedidas em suas empreitadas precisam atender interesses mais complexos e, conforme mencionado, a questão de sustentabilidade empresarial passa a ser preocupação essencial para a Governança Corporativa. Alguns estudos internacionais mostraram que há uma relação positiva entre internacionalização de empresas e práticas sustentáveis. Destaca-se o estudo de Kolk e Van Tulder (2010) que investigaram que, ao mesmo tempo em que as multinacionais são parte dos problemas ambientais e sociais existentes, elas também são parte da solução, tendo em vista que as instituições de países mais exigentes acabam por influenciar a atuação de empresas internacionais em países com menor preocupação sustentável. Além disso, cabe ressaltar que neste estudo também se tomou como base os preceitos da Teoria dos Stakeholders.

Chakrabarty e Wang (2012), por meio de uma análise com dados em painéis, no período de 1989 a 2009, verificaram que multinacionais que têm uma combinação de alta intensidade em pesquisa e desenvolvimento e alta internacionalização são mais prováveis a desenvolverem práticas de sustentabilidade e possuem maior chance de manter tais práticas a longo prazo. Destaca-se também o trabalho de Attig, Boubakri, Ghoul e Guedhami (2016), os quais estudaram empresas dos Estados Unidos e o efeito da internacionalização em suas práticas sustentáveis. Para isso, utilizou-se informações de 3.040 empresas no período 19912010. Os resultados, obtidos a partir de uma análise de regressão linear, mostram que a internacionalização apresenta um efeito positivo 
sobre as práticas de responsabilidade social corporativa.

Estudos mais recentes também evidenciaram essa relação. Hörisch, Burritt, Christ e Schaltegger (2017) compararam a influência de diferentes sistemas jurídicos nas práticas de gestão de sustentabilidade corporativa em relação à crescente internacionalização das atividades empresariais. Foram estudados três sistemas legais e feita uma análise comparativa por meio de regressão de mais de 200 grandes corporações em cinco países: direito comum (EUA e Austrália), lei de código alemão (Alemanha) e código francês (França e Espanha). Descobriu-se que o grau de internacionalização consegue moderar a influência do sistema jurídico nas práticas corporativas de sustentabilidade.

Já na pesquisa realizada por Symeou, Zyglidopoulos e Williamson (2017), os resultados mostraram que a internacionalização impactou positivamente as práticas sustentáveis de empresas extrativas de recursos naturais. Os autores evidenciaram que a exigência de licenças em certos países aumentou o nível de preocupação sustentável dessas organizações.

Por fim, cabe ressaltar o estudo nacional de Soschinski, Brandt e Klann (2019), os quais analisaram a influência da internacionalização nas práticas de responsabilidade social corporativa (RSC) em empresas brasileiras.

Os resultados apontaram que o nível de internacionalização das empresas se mostrou positivamente relacionado à RSC, mas apenas em sua dimensão social e para empresas não reguladas. Em linhas gerais, os autores concluíram que a busca por ações de RSC por parte das empresas brasileiras tem relação com sua inserção no mercado internacional, como forma de atender possíveis pressões de stakeholders.

Destarte, tomando-se como base a análise multidimensional da internacionalização empresarial (internacionalização do capital social, das receitas e dos mercados), à luz da Teoria dos Stakeholders e com base nas recomendações da literatura, levantase a seguinte hipótese geral a ser testada:

H1 - Há uma relação positiva entre a internacionalização e a sustentabilidade empresarial no Brasil.

\section{PROCEDIMENTOS METODOLÓGICOS}

Quanto aos objetivos, esta pesquisa classifica-se como descritiva (Collis \& Hussey, 2005) por delinear as características de determinado grupo de empresas e por estabelecer correlações entre suas duas variáveis: internacionalização e sustentabilidade empresarial. No que diz respeito ao problema, caracteriza-se como quantitativa, com abordagem empírico-analítica, adotando-se o emprego de instrumentos estatísticos (Sampieri, Collado \& Lúcio, 2013). Quanto à coleta dos dados, trata-se de pesquisa documental, por utilizar dados e materiais não editados (Martins \& Theóphilo, 2009).

A população da pesquisa reúne as 100 empresas listadas na B3 S.A. com maior valor de mercado, de acordo com dados extraídos do Economática ${ }^{\circledR}$. Após exclusão de cinco empresas que não apresentaram os dados necessários para a pesquisa, a amostra ficou definida em 95 empresas. Foram utilizados dados secundários oriundos das Demonstrações Financeiras Padronizadas (DFPs) e do Formulário Referência (FR) das empresas, disponibilizados no website da B3 S.A., além de informações contidas em Índices e Rankings de Sustentabilidade. Os dados referem-se ao exercício social de 2017, sendo coletados em novembro de 2018.

Especificamente na etapa de coleta dos dados, inicialmente recorreu-se à análise de conteúdo, aplicada aos documentos das empresas, em especial ao Formulário de Referência, de onde se extraiu os dados de internacionalização, demandados nesta análise. Com a análise de conteúdo, informações suplementares são fornecidas ao pesquisador, que percorre as fases de pré-análise (seleção do material), exploração do material (documentos), tratamento dos resultados, inferência e interpretação (Bardin, 2011).

Para atender ao objetivo proposto, a amostra foi distribuída em dois grupos: a) empresas sustentáveis, com base na participação em pelo menos um dos Índices Bovespa - ISE e ICO2 - (Carvalhal \& Tavares, 2013; Lameira, Ness, Quelhas \& Pereira, 2013; Guimarães, Peixoto \& Carvalho, 2017) ou rankings de sustentabilidade (edições de 2017), a saber: 1) Guia Exame de Sustentabilidade, 2) Ranking Imprensa - As Empresas Mais Sustentáveis Segundo a Mídia, 3) Ranking Merco - Monitor Empresarial de Reputação Corporativa, 4) Guia Exame Maiores e Melhores Empresas Brasileiras e 5) 150 Melhores Empresas Para Trabalhar - Você S.A.; e b) empresas não 
sustentáveis. A escolha dos índices e rankings se deu pelas distintas perspectivas de análise, os quais compreendem pelo menos uma das dimensões de sustentabilidade - ambiental, econômica e social (Davies, Chun, Silva \& Roper, 2003). Destarte, a sustentabilidade empresarial de cada empresa é mensurada a partir de sua participação em pelo menos um dos dois índices ou um dos cinco rankings mencionados, sendo estes os principais índices e rankings utilizados em pesquisas anteriores (Cardoso, De Luca \& Gallon, 2014; Lima, Domingos, Vasconcelos \& Rebouças, 2015; Domencio, Mazzioni, Magro, Peruzzo \& Peruzzo, 2015; Lopes, De Luca,
Góis \& Vasconcelos, 2017) validando, portanto, a sua utilização nesta pesquisa.

A variável dependente (sustentabilidade empresarial) apresenta-se como dicotômica (dummy), na qual foi atribuído o valor 0 (zero) para empresas não sustentáveis - as que não possuem participação em índices e/ou rankings de sustentabilidade - e 1 (um) para empresas sustentáveis.

As variáveis independentes, constituintes da internacionalização, contidas no modelo, suas métricas, a fonte de coleta dos dados e a base teórica para cada uma delas estão explícitas na Tabela 1.

Tabela 1 Variáveis independentes da internacionalização

\begin{tabular}{|l|l|l|l|}
\hline \multicolumn{1}{|c|}{ Variável } & \multicolumn{1}{|c|}{ Métrica } & Fonte & \multicolumn{1}{c|}{ Fundamentação } \\
\hline $\begin{array}{l}\text { Internacionalização } \\
\text { do capital social }\end{array}$ & $\begin{array}{l}\text { Participação de acionistas } \\
\text { estrangeiros no capital social }\end{array}$ & & $\begin{array}{l}\text { Maia, Vasconcelos e De Luca (2013) } \\
\text { Santos, Vasconcelos e De Luca (2015) }\end{array}$ \\
\cline { 1 - 2 } $\begin{array}{l}\text { Internacionalização } \\
\text { das receitas }\end{array}$ & $\begin{array}{l}\text { Proporção de receitas } \\
\text { provenientes do exterior, em } \\
\text { relação ao faturamento total }\end{array}$ & $\begin{array}{l}\text { Formulário de } \\
\text { Referência }\end{array}$ & $\begin{array}{l}\text { Floriani e Fleury (2012) Ruigrok e } \\
\text { Wagner (2003) Sullivan (1994) }\end{array}$ \\
\cline { 1 - 2 } $\begin{array}{l}\text { Internacionalização } \\
\text { dos mercados }\end{array}$ & $\begin{array}{l}\text { Número de países onde a } \\
\text { empresa possui instalações }\end{array}$ & & $\begin{array}{l}\text { Floriani e Fleury (2012) Ruigrok e } \\
\text { Wagner (2003) }\end{array}$ \\
\hline
\end{tabular}

Fonte: Elaborada pelos autores.

No presente estudo foram ainda incrementadas algumas medidas de controle para neutralizar efeitos que também podem impactar a análise. Tais variáveis são: Tamanho (logarítimo natural do Ativo Total) e Endividamento (Exigível/Patrimônio Líquido), obtidas a partir de dados coletados na base Economática ${ }^{\circledR}$; Setor de atuação, Participação no Novo Mercado (NM) e Presença de Comitê de Sustentabilidade, obtidas a partir do FR da B3 S.A. e Carteiras do Índice de Governança Corporativa (Carvalhal da Silva \& Chien, 2013; Lameira et al., 2013; Cardoso, De Luca \& Gallon, 2014; Guimarães, Peixoto \& Carvalho, 2017; Lopes, De Luca, Góis \& Vasconcelos, 2017).

Com o intuito de responder à questão suscitada nesta investigação e de atender ao objetivo do estudo, os dados foram analisados por meio das seguintes técnicas estatísticas: Teste de Diferença entre Médias e Regressão Logística.

Para atingir o objetivo geral do estudo, inicialmente, utilizou-se da estatística descritiva, com a indicação de máximos e mínimos, média e desviospadrão. Ressalta-se, também, a utilização do teste de diferença de média de Mann-Whitney (duas amostras independentes), a fim de verificar a existência de diferenças significativas, estatisticamente, das variáveis utilizadas entre as empresas sustentáveis e não sustentáveis, quanto à internacionalização. Foi utilizado o teste de MannWhitney, pois os testes Kolmogorov-Smirnov e Shapiro Wilk verificaram que os dados não apresentavam distribuição normal.

Em seguida, para verificar quais as variáveis que mais impactam na probabilidade de as empresas serem mais sustentáveis, utilizou-se a análise de Regressão Logística, que consiste em uma ferramenta de análise multivariada que tem por objetivo segregar grupos (variável dependente) considerando os efeitos das variáveis independentes na discriminação dos casos observados (Minussi, Damacena \& Ness Jr, 2002). De acordo com Dias Filho e Corrar (2009) a regressão logística tem algumas particularidades que a distingue dos demais modelos de regressão. A principal é a dicotomia que possui a variável dependente, exigindo que o resultado das análises se associe às categorias, como aceitar ou rejeitar, positivo ou negativo. Destaca-se ainda que os resultados da variável dependente devem permitir interpretações em termos de probabilidade e não apenas classificação em categorias (Dias Filho \& 
Corrar, 2009). Assim, foi empregado o seguinte modelo nesta pesquisa (Equação 1):

logit (SUST) $=B_{0}+B_{1}$ INTC + $B_{1}$ INTR + $B_{1}$ INTM +

$B_{2} T A M+B_{3} E N D V+B_{4} S T+B_{5} N M+B_{6} C S+\varepsilon$

Em que, SUST: Sustentabilidade Empresarial; INTC: Internacionalização do capital social; INTR: Internacionalização das receitas; INTM: Internacionalização dos mercados; TAM: Tamanho; ENDV: Endividamento; ST: Setor de atuação; NM: Participação no Novo Mercado; CS: Presença de Comitê de Sustentabilidade e $\varepsilon$ : Termo de Erro.
As análises foram processadas com o auxílio dos pacotes estatísticos Statistic Package for Social Sciencies (SPSS) - versão 22.0 e Stata - versão 13.

\section{ANÁLISE DOS RESULTADOS}

Para o alcance do objetivo do estudo - investigar a relação entre a internacionalização e a sustentabilidade empresarial em companhias listadas na B3 S.A. -, inicialmente, na Tabela 2, encontra-se evidenciada a representatividade da internacionalização nas 95 empresas da amostra, bem como a estatística descritiva das variáveis de internacionalização.

Tabela 2 Estatística descritiva das variáveis de internacionalização

\begin{tabular}{l|c|c|c|c|c}
\hline \multicolumn{1}{c|}{ Variável } & $\begin{array}{c}\text { No de } \\
\text { empresas }\end{array}$ & Média & Desvio-padrão & Mínimo & Máximo \\
\hline Capital Social & 51 & $20,3 \%$ & 19,08 & $2,2 \%$ & $72,3 \%$ \\
Mercados & 30 & 9,8 & 11,61 & 1 & 64 \\
Receitas & 36 & $36,9 \%$ & 32,95 & $0,0 \%$ & $92,5 \%$ \\
\hline
\end{tabular}

Fonte: elaborada pelos autores.

A análise descritiva das variáveis que representam a internacionalização apresentou que as maiores empresas em valor de mercado, de capital aberto do Brasil, listadas na bolsa, em sua maioria internacionalizaram-se através do capital. São 51 empresas com participação estrangeira no capital, com uma média de aproximadamente $20 \%$. Apenas um terço da amostra, aproximadamente, internacionalizou-se, exportando ou se instalando em outros países.

Esse achado certifica a afirmação de Smaniotto, Paiva e Vieira (2012) de que a temática sobre internacionalização é recente no cenário empresarial brasileiro, já que, diante dos resultados, a internacionalização das empresas pode ser verificada pelos processos menos complexos, apresentados pela participação de investidores estrangeiros no capital social e pelas vendas ao mercado.

No que diz respeito à internacionalização dos mercados, somente 30 empresas possuem subsidiárias em outros países, com uma média de 9 países por empresa. Com subsidiárias em 64 países, a Dufry $A G$ é a empresa com o mais alto nível de dispersão geográfica, conforme identificado em seu Formulário de Referência.

No que tange à internacionalização das receitas, são 36 as empresas que exportam, com uma média de aproximadamente 37\%. Esse resultado diverge da pesquisa de Floriani e Fleury (2012), os quais afirmam que a exportação é a forma mais comum de internacionalização. Os dados apresentaram elevada dispersão, com alta variabilidade entre as empresas da amostra, destacando-se a Vale com as receitas provenientes de subsidiárias estrangeiras correspondendo a $92 \%$ do faturamento total.

A Tabela 3 apresenta a estatística descritiva das variáveis de internacionalização agrupadas pela variável dummy, que classifica as empresas da amostra como sustentáveis e não sustentáveis. 
Tabela 3 Estatística descritiva das variáveis das empresas da amostra

\begin{tabular}{l|l|c|c|c|c}
\hline \multicolumn{2}{c|}{ Variável } & Média & Desvio-padrão & Mínimo & Máximo \\
\hline $\begin{array}{l}\text { Internacionalização } \\
\text { do Capital Social }\end{array}$ & Sustentáveis & 10,89 & 15,49 & 0,00 & 61,88 \\
\hline Internacionalização & São Sustentáveis & 12,91 & 20,68 & 0,00 & 72,32 \\
Mercados & Não Sustentáveis & 3,87 & 6,10 & 0 & 26 \\
\hline Internacionalização & 2,51 & 9,64 & 0 & 64 \\
Receitas & Sustentáveis & 8,47 & 10,83 & 0,00 & 25,70 \\
\hline \multirow{2}{*}{ Tamanho } & Não Sustentáveis & 6,71 & 9,17 & 0,00 & 22,41 \\
\hline \multirow{2}{*}{ Endividamento } & Sustentáveis & 23,64 & 1,26 & 21,14 & 27,41 \\
& Não Sustentáveis & 22,39 & 1,19 & 17,72 & 24,72 \\
\hline
\end{tabular}

Fonte: Elaborada pelos autores.

Os achados indicam que as empresas sustentáveis apresentam maior grau de internacionalização, exceto para a dimensão do capital social. As empresas da amostra que se internacionalizam através da instalação em outros países e através da exportação apresentam-se, em média, como mais sustentáveis. Embora o maior número de instalações esteja nas empresas não sustentáveis, a média das empresas sustentáveis é superior, indicando que o número máximo não é capaz de caracterizar o grupo. Destaca-se também que as empresas sustentáveis, também são as maiores empresas e as mais endividadas conforme indica a descrição de mínimo e máximo.

Para analisar diferenças significativas entre as variáveis das empresas sustentáveis e não sustentáveis, aplicou-se o teste de diferença de médias para amostras independentes de MannWhitney, conforme Tabela 4. Através dos testes de Kolmogorov-Smirnov e Shapiro-Wilk, certificou-se que quase todas as variáveis não apresentaram distribuição normal, pois o p-valor dos testes foi inferior a 0,05, assim justificando a utilização do teste não paramétrico.

Tabela 4 Teste de Mann-Whitney

\begin{tabular}{|c|c|c|c|c|c|c|}
\hline \multicolumn{2}{|c|}{ Internacionalização } & \multirow{2}{*}{$\begin{array}{c}\begin{array}{c}\text { № de } \\
\text { empresas }\end{array} \\
48 \\
47 \\
\end{array}$} & \multirow{2}{*}{$\begin{array}{c}\text { Mann- } \\
\text { Whitney }\end{array}$} & \multirow{2}{*}{$\begin{array}{c}\text { Wilcoxon W } \\
2191,5\end{array}$} & \multirow{2}{*}{$\begin{array}{c}Z \\
-0,499\end{array}$} & \multirow{2}{*}{$\begin{array}{c}\text { Asymp. } \\
\text { Sig. } \\
\text { (2-tailed) } \\
0,618\end{array}$} \\
\hline Capital Social & $\begin{array}{l}\text { Sustentáveis } \\
\text { Não Sustentáveis }\end{array}$ & & & & & \\
\hline Mercados & $\begin{array}{l}\text { Sustentáveis } \\
\text { Não Sustentáveis }\end{array}$ & $\begin{array}{l}48 \\
47 \\
\end{array}$ & 913,0 & 2041,0 & $-1,941$ & $0,050^{*}$ \\
\hline Receitas & $\begin{array}{l}\text { Sustentáveis } \\
\text { Não Sustentáveis }\end{array}$ & $\begin{array}{l}48 \\
47\end{array}$ & 993,0 & 2121,0 & $-1,152$ & 0,249 \\
\hline
\end{tabular}

Nota: $\left({ }^{*}\right)$ Significante a $5 \%$.

Fonte: Elaborada pelos autores.

Com base nos resultados apresentados na Tabela 4, pode-se constatar que apenas uma das três dimensões de análise da internacionalização apontou diferença significativa na comparação entre as empresas sustentáveis e não sustentáveis: a internacionalização de mercados.

Desse modo, foi delineada a regressão logística, com o intuito de se comprovar a hipótese geral de que há uma relação positiva entre a internacionalização e a sustentabilidade empresarial no Brasil. Na Tabela 5, dispõem-se os resultados da regressão, provenientes da Equação 1 em dois modelos. No modelo 1 as variáveis de internacionalização foram contínuas, já no modelo 2 as variáveis foram estimadas a partir de dummy. As variáveis dummies foram definidas da seguinte forma: atribuiu-se o valor 0 (zero) para as empresas não sustentáveis, bem como para as empresas não internacionalizadas; e 1 (um) para as empresas sustentáveis, como também para as empresas internacionalizadas, nas três dimensões analisadas (capital social, receitas e mercados). 
Tabela 5 Regressão Logística Sustentabilidade Empresarial

\begin{tabular}{l|c|c|c|c|c|c|c|c}
\hline \multirow{4}{*}{ Variável } & \multicolumn{4}{|c|}{ Modelo 1 } & \multicolumn{4}{c}{ Modelo 2 } \\
\cline { 2 - 10 } & LR Qui & Pseudo R & Sig. & N & LR Qui $^{2}$ & $\begin{array}{c}\text { Pseudo } \\
\mathbf{R}^{2}\end{array}$ & Sig. & N \\
\cline { 2 - 10 } & 36,72 & 0,2788 & 0,000 & 95 & 38,73 & 0,2941 & 0,000 & 95 \\
\cline { 2 - 10 } & Coef. & $\begin{array}{c}\text { Erro } \\
\text { padrão }\end{array}$ & $\mathbf{Z}$ & Sig. & Coef. & $\begin{array}{c}\text { Erro } \\
\text { padrão }\end{array}$ & $\mathbf{Z}$ & Sig. \\
\hline Inter_Capital & $-0,031$ & 0,018 & $-1,74$ & $0,082^{* * *}$ & 0,945 & 0,979 & 0,97 & 0,334 \\
\hline Inter_Mercados & $-0,017$ & 0,034 & $-0,52$ & 0,601 & 1,934 & 1,034 & 1,87 & $0,061^{* * *}$ \\
\hline Inter_Receitas & 0,024 & 0,035 & 0,63 & 0,488 & $-0,833$ & 1,018 & $-0,82$ & 0,413 \\
\hline Tamanho & 1,036 & 0,319 & 3,24 & $0,001^{*}$ & 0,858 & 0,293 & 2,93 & $0,003^{*}$ \\
\hline Endividamento & 0,122 & 0,079 & 1,54 & 0,123 & 0,129 & 0,088 & 1,47 & 0,143 \\
\hline Setor de Atuação & $-1,324$ & 0,874 & $-1,51$ & 0,130 & $-1,194$ & 0,817 & $-1,46$ & 0,144 \\
\hline Comitê de Sust. & 2,019 & 0,940 & 2,15 & $0,032^{* *}$ & 1,863 & 0,942 & 1,98 & $0,048^{* *}$ \\
\hline Novo Mercado & $-0,287$ & 0,542 & $-0,53$ & 0,597 & $-0,400$ & 0,553 & $-0,72$ & 0,470 \\
\hline Constante & $-24,35$ & 7,332 & $-3,32$ & $0,001^{*}$ & $-20,24$ & 6,684 & $-3,03$ & $0,002^{*}$ \\
\hline
\end{tabular}

Nota: $\left({ }^{*}\right)$ Significante a $1 \%(* *)$ Significante a $5 \%(* * *)$ Significante a $10 \%$.

Fonte: Elaborada pelos autores.

Na Tabela 5, constata-se que o poder explicativo, baseado no Pseudo $R^{2}$ dos modelos 1 e 2 são de $27,88 \%$ e $29,41 \%$ respectivamente, e ambos são significantes ao nível de $1 \%$. Desse modo, os dois modelos são válidos e pode-se inferir resultados.

Observa-se que em ambos os modelos, a internacionalização através das receitas não indica relação com a sustentabilidade empresarial. No modelo 1 , somente a variável de internacionalização através do capital social apresentou relação significativa com a sustentabilidade, todavia, essa relação foi negativa. A relação negativa entre as variáveis indica que as empresas com menor grau de composição estrangeira em seu capital social são mais sustentáveis. Considera-se tal achado como inesperado, uma vez que contrapõe a hipótese levantada nesse estudo, de que há uma relação positiva entre a internacionalização e a sustentabilidade empresarial nas empresas brasileiras. No modelo 2, a variável internacionalização de mercados apresentou relação positiva e significante com as práticas sustentáveis, ou seja, as empresas que possuem alta dispersão geográfica são mais sustentáveis. Esse achado corrobora com estudos internacionais que apontam essa relação positiva (Kolk \& Van Tulder, 2010, Attig, Boubakri, Ghoul \& Guedhami, 2016 Chakrabarty \& Wang, 2012; Hörisch, Burritt, Christ \& Schaltegger, 2017, Symeou, Zyglidopoulos \& Williamson, 2017), permitindo-se explorar algumas considerações.
Instalar unidades em outros países expõe as empresas a um nível mais complexo de internacionalização, uma vez que estas unidades terão um relacionamento direto com uma maior gama de stakeholders. Na literatura, existe um consenso de que as empresas se engajam práticas sustentáveis como resposta a pressões sociais de stakeholders, que podem ser exercidas de diferentes formas: reivindicações da comunidade relacionadas a questões ambientais (Kassinis \& Vafeas, 2006); exigências de consumidores (Besley \& Ghatak, 2007); pressões da estrutura de mercado competitiva (Fisman, lyengar, Kamenica \& Simonson, 2008); e pressões de organizações não governamentais e da sociedade (Baron, 2009).

Desse modo, é fundamental que as empresas adotem processos de envolvimento dos stakeholders para estabelecer e aprimorar sua legitimidade para operar, e, consequentemente, sua viabilidade e continuidade (Michelon \& Parbonneti, 2010). Entre esses processos, destaca-se a sustentabilidade empresarial, considerando-se tanto as legislações locais como as culturas dos países participantes dessa descentralização geográfica, e tendo em vista que as instituições de países mais exigentes acabam por influenciar a atuação de empresas internacionais em países com menor preocupação sustentável. Dessa forma, a Teoria dos Stakeholders mostra-se consistente na hipótese do alívio de pressões, explicando que as empresas empreendem atividades 
Internacionalização e Sustentabilidade Empresarial no Brasil

socioambientais para mitigar pressões exercidas por stakeholders.

Além disso, cabe ressaltar que no cenário de economia global e com a internacionalização de investimentos, as práticas de sustentabilidade corporativa cada vez mais adquirem relevância, pois à medida do sucesso da introdução de práticas de sustentabilidade empresarial, a empresa muda sua forma de atuar, de tomar decisões e, por definição a sua contribuição e a sua relação com o mercado e a sociedade em que atua, conforme mencionam Benites e Polo (2013).

Entretanto, a hipótese de pesquisa de que há uma relação positiva entre a internacionalização e a sustentabilidade empresarial nas firmas brasileiras foi rejeitada, tendo em vista que somente a dimensão internacionalização de mercados apresentou relação positiva e significante com a sustentabilidade corporativa. Dessa forma, pode se concluir que, no mercado brasileiro, os resultados sobre a relação entre sustentabilidade empresarial e internacionalização são inconclusivos, pois, a depender da dimensão de internacionalização em análise, os achados podem ser distintos. Ressalte-se que, nesse estudo, optou-se por uma análise multidimensional da internacionalização, com o objetivo de captar o efeito dessa estratégia empresarial a partir de diversas perspectivas.

\section{CONSIDERAÇÕES FINAIS}

No contexto das empresas de capital aberto listadas na B3 S.A., com maior valor de mercado segundo dados do Economática ${ }^{\circledR}$, investigou-se a relação entre a internacionalização e a sustentabilidade empresarial, à luz da Teoria dos Stakeholders. Tratando-se a internacionalização sob múltiplas dimensões, procurou-se suprir a lacuna existente nas pesquisas nacionais acerca da relação entre os dois construtos.

Por meio de análise descritiva, verificou-se que a internacionalização atinge diferentemente as empresas, em proporção e intensidade. Os aspectos que mais influenciam as empresas ao se internacionalizar são o capital social, as exportações e a presença física (instalação) em outros países, nessa ordem de importância. Segundo a literatura, este último acarreta mais risco e demanda mais comprometimento da empresa, o que pode justificar o resultado encontrado. Todavia, a exportação, apontada como meio mais frequente de internacionalização, não apresentou resultados convergentes com os de outros estudos. Ainda por meio da análise descritiva, verificou-se, através da média e da medida de máximo, que as empresas sustentáveis possuem um maior grau de internacionalização por meio do capital social e da dispersão geográfica.

O resultado do teste de diferenças entre médias revelou que apenas as empresas internacionalizadas por meio da presença física em outros países apresentaram diferenças estatisticamente significantes no tocante à sustentabilidade empresarial. Com a aplicação da regressão logística, identificou-se a relação positiva entre a sustentabilidade empresarial e a internacionalização apenas quando está mensurada através da presença de instalações em outros países. As demais variáveis não apresentaram relação significante.

Dessa forma, rejeita-se a hipótese de pesquisa que há uma relação positiva entre a internacionalização e a sustentabilidade empresarial no Brasil. Entretanto, destaca-se que os achados apresentam algumas limitações, suscitando implicações que podem ser levantadas para os achados inesperados. O presente estudo cobre apenas um período, podendo ser replicado em um lapso temporal maior, suscitando inferência acerca do efeito temporal dos dados. Destaca-se também a utilização de participação em índices e rankings de sustentabilidade como proxy para essa temática, uma vez que a utilização da dummy impossibilita a mensuração das práticas de sustentabilidade.

Entende-se que o estudo da internacionalização e da sustentabilidade empresarial proporciona reflexões sobre a busca pelo valor a longo prazo, permitindo assim visualizar a Teoria dos Stakeholders, a qual sustenta que as empresas, para garantir valor a longo prazo, necessitam conciliar os interesses dos diversos stakeholders envolvidos, como acionistas, empregados, clientes, fornecedores e comunidade. Ressalta-se, portanto, a importância da sustentabilidade empresarial como estratégia para a melhoria da imagem e reputação das empresas, captação vantajosa de recursos e maior vantagem competitiva.

A temática demonstra-se relevante tanto para o meio acadêmico como para o ambiente empresarial. No contexto científico, o estudo apresenta uma abordagem metodológica multidimensional que 
permitiu a análise da estratégia de internacionalização sobre múltiplas perspectivas (capital social, mercados e receitas) para a investigação proposta. Para o mercado, é notória a crescente discussão sobre a sustentabilidade empresarial e quais são os fatores que corroboram para essas práticas no ambiente corporativo. Entender a internacionalização como parte desse processo é fundamental para as empresas que buscam crescimento, competitividade e continuidade, tendo em vista o engajamento com os seus diversos stakeholders.

Há uma escassez de estudos acerca dessa temática, e as pesquisas encontradas são incipientes, havendo, portanto, a necessidade de maior aprofundamento no assunto. A pesquisa avança nos campos teórico e prático, porém algumas limitações

\section{REFERÊNCIAS}

Albuquerque Filho, A., Freire, M., De Luca, M., \& Vasconcelos, A. (2020). Influência da internacionalização e da inovação na competitividade empresarial. Internext, 15(1), 01-18. DOI: http://dx.doi.org/10.18568/internext.v15i1.521

Attig, N., Boubakri, N., El Ghoul, S., \& Guedhami, O. (2016). Firm internationalization and corporate social responsibility. Journal of Business Ethics, 134(2), 171-197. DOI: https://doi.org/10.1007/s10551-014-2410-6

Bardin, L. (2011). Análise de conteúdo. Lisboa: Edições 70.

Baron, D. P. (2009). A positive theory of moral management, social pressure, and corporate social performance. Journal of Economics \& Management Strategy, 18(1), 7-43.

Benites, L. L. L., \& Polo, E. F. (2013). A sustentabilidade como ferramenta estratégica empresarial: Governança Corporativa e aplicação do Triple Bottom Line na Masisa. Revista de Administração UFSM, 6(1), 195-210. DOI: http://dx.doi.org/10.5902/198346598879

Besley, T., \& Ghatak, M. (2007). Retailing public goods: The economics of corporate social responsibility. Journal of public Economics, 91(9), 1645-1663. podem ser apontadas, razão pela qual sugere-se uma reflexão para futuras pesquisas, tanto quanto à internacionalização corporativa como em relação à sustentabilidade empresarial, em especial quanto ao uso de outras medidas e ampliação de variáveis. Para além, recomenda-se uma amostra que contemple todas as empresas de capital aberto listadas na bolsa brasileira, bem como a análise das economias envolvidas na internacionalização das empresas.

Destaca-se, ainda, a possibilidade de análise comparativa entre empresas de países em desenvolvimento e empresas de nações desenvolvidas. Por fim, sugere-se a realização de uma pesquisa qualitativa com as empresas da amostra, no intuito de esclarecer o porquê da relação inconclusiva entre os constructos, como também analisar se as políticas públicas exercem efeito nesta relação.

Bouças, A. S., \& Gomes, J. S. (2010). Controle gerencial em empresas brasileiras internacionalizadas: o caso de uma empresa de material elétrico. Revista Universo Contábil, 6(2), 626. DOI:10.4270/ruc. 2010210

B3 S.A. - Brasil, Bolsa, Balcão. Índice de Carbono Eficiente (ICO2). Recuperado de: http://www.bmfbovespa.com.br/pt_br/produtos/in dices/indices-de-sustentabilidade/indice-carbonoeficiente-ico2.htm., em 20 nov. 2017.

Cardoso, V. I. C., De Luca, M. M. M., \& Gallon, A. V. (2014). Reputação corporativa e o disclosure socioambiental de empresas brasileiras. Contabilidade, Gestão e Governança, 17(2), 26-44.

Carvalhal, A., \& Tavares, E. (2013). Does social responsability enhance firm value and return in Brazil? Corporate Ownership \& Control, 10(2), 253258.

Carvalhal da Silva, A. L. \& Chien, A. C. Y. (2013). Remuneração executiva, valor e desempenho das empresas brasileiras listadas. Revista Brasileira de Finanças, 11(4), 481-502.

Chakrabarty, S., \& Wang, L. (2012). The long-term sustenance of sustainability practices in MNCs: A dynamic capabilities perspective of the role of $R \& D$ and internationalization. Journal of Business Ethics, 110(2), 205-217. DOI: 10.1007/s10551-012-1422-3 
Clarkson, M. E. (1995). A stakeholder framework for analyzing and evaluating corporate social performance. Academy of management review, 20(1), 92-117.

Collis, J., \& Hussey, R. (2005). Pesquisa em administração: um guia prático para alunos de graduação e pós-graduação. Tradução Lúcia Simonini. Porto Alegre: Bookman.

Cuervo-Cazurra, A. (2012). How the analysis of developing country multinational companies helps advance theory: solving the goldilocks debate. Global Strategy Journal, 2(3), 153-167.

Cuervo-Cazurra, A., Maloney, M., \& Manrakhan, S. (2007). Causes of the difficulties in internationalization. Journal of International Business Studies, 38(5), 709-725. DOI: 10.1057/palgrave.jibs. 8400295

Davies, G., Chun, R., Da Silva, R.V., \& Roper, S. (2003). Corporate Reputation and Competitiveness. Corporate Communications: An International Journal, 8 (2), 48-149.

Denberg, M. W. S., \& Gomes, J. S. (2011). Impactos ocasionados pela internacionalização em empresas brasileiras: um estudo de caso múltiplo. Contabilidade, Gestão e Governança, 14(3), 77-99. DOI: 10.4270/RUC.2013210

Dias Filho J. M. \& Corrar, L. J. (2009). Regressão logística. In: Corrar, L. J., Paulo, E. \& Dias Filho, J. M. (Org.). Análise multivariada: para os cursos de administração, ciências contábeis e economia. São Paulo: Atlas.

Domenico, D. D., Mazzioni, S., Magro, C. B. D., Peruzzo, M., \& Peruzzo, M. (2015). Análise dos indicadores ambientais das empresas listadas no Guia de Sustentabilidade da Revista Exame. Ciências Sociais Aplicadas em Revista, 15(28), 65-89.

Donaldson, T., \& Preston, L. E. (1995). The stakeholder theory of the corporation: concepts, evidence and implications. Academy of Management Review, 20(1), 65-91. DOI: 10.2307/258887

Dyllick T., \& Hockerts K. (2002). Beyond the business case for corporate sustainability. Business
Strategy and the Environment, 11, 130-141. DOI: 10.1002/bse.323

Elkington, J. (2001). Canibais com garfo e faca. São Paulo: Makron Books.

Fisman, R., Iyengar, S. S., Kamenica, E., \& Simonson, I. (2008). Racial preferences in dating. The Review of Economic Studies, 75(1), 117-132.

Floriani, D. E., \& Fleury, M. T. (2012). O efeito do grau de internacionalização nas competências internacionais e no desempenho financeiro da PME brasileira. Revista de Administração Contemporânea - RAC, 16(3), 438-458. DOI:

\section{http://dx.doi.org/10.1590/S1415-}

\section{7}

Freeman, R. E. (1984). Strategic management: A stakeholder approach. Boston: Pitman.

Freeman, R., \& Mcvea, J. (2001). A stakeholder approach to strategic management. The Blackwell handbook of strategic management, 189-207.

Furlan, F. (2013). O sinal que vem das ruas. Guia Exame de Sustentabilidade 2013. São Paulo: Editora Abril.

Guimarães, T. M., Peixoto, F. M., \& Carvalho, L. (2017), Sustentabilidade empresarial e governança corporativa: uma análise da relação do ISE da BM\&FBovespa com a compensação dos gestores de empresas brasileiras. Revista de Educação e Pesquisa em Contabilidade, 11(2), 134-149. DOI: https://doi.org/10.17524/repec.v11i2.1418

Guay, T., Doh, J. P., \& Sinclair, G. (2004). Nongovernmental organizations, shareholder activism, and socially responsible investments: Ethical, strategic, and governance implications. Journal of Business Ethics, 52(1), 125-139. DOI: 10.1023/B:BUSI.0000033112.11461.69

Hörisch, J., Burritt, R. L., Christ, K. L. \& Schaltegger, S. (2017). Legal systems, internationalization and corporate sustainability. An empirical analysis of the influence of national and international authorities. Corporate Governance: The International Journal of Business in Society, 17(5), 861-875. DOI: https://doi.org/10.1108/CG-08-2016-0169 
Irene, M. H., \& Robin, E. R. (2002). Developing Awareness of the Sustainability Concept. The Journal of Environmental Education, 34(1), 16-20. DOI: https://doi.org/10.1080/00958960209603477

Jensen, M. (2001). Value Maximization, Stakeholder Theory, and the Corporate Objective Function. Journal of Applied Corporate Finance, 14(3), 8-21. DOI: $\quad$ https://doi.org/10.1111/j.17456622.2001.tb00434.x

Kacperczyk, A. (2009). With greater power comes greater responsibility? Takeover protection and corporate attention to stakeholders. Strategic Management Journal, 30, 261-285. DOI: https://doi.org/10.1002/smj. 733

Kang, J. (2013). The relationship between corporate diversification and corporate social performance. Strategic Management Journal, 34, 94109. DOI: https://doi.org/10.1002/smi.2005

Kassinis, G., \& Vafeas, N. (2006). Stakeholder pressures and environmental performance. Academy of Management Journal, 49(1), 145-159.

Kim, Y., \& Kim, S. Y. (2010). The influence of cultural values on perceptions of corporate social responsibility: Application of Hofstede's dimensions to Korean public relations practitioners. Journal of Business Ethics, 91(4), 485-500. https://doi. 10.1007/s10551-009-0095-z

Knoepfel, I. (2001). Dow Jones Sustainability Group Index: a global benchmark for corporate sustainability, Corporate Environmental Strategy, 8(1), 6-15. DOI: 10.1016/S1066-7938(00)00089-0

Kolk, A. \& Van Tulder, R. (2010). International business, corporate social responsibility and sustainable development. International Business Review, V.19, 2, $119-125 . \quad$ DOI: http://dx.doi.org/10.1016/j.ibusrev.2009.12.003

Lameira, V. J., Ness Jr., W, L., Quelhas, O, L, G., \& Pereira, R, G. (2013). Sustainability, value, performance and risk in the Brazilian capital markets. Revista Brasileira de Gestão e Negócios, 15(46), 76 90. DOI: http://dx.doi.org/10.7819/rbgn.v15i46.1302

Lima, L. C., Domingos, S. R. M., Vasconcelos, A. C., \& Rebouças, S. M. D. P. (2015). Reputação e qualidade da governança corporativa das companhias abertas brasileiras. Revista de Administração FACES Journal, 14(2), 8-43. DOI: http://dx.doi.org/10.21714/19846975FACES2015V14N2ART2034

Lopes, A. C., De Luca, M. M. M., Góis, A. D., \& de Vasconcelos, A. C. (2017). Disclosure socioambiental, reputação corporativa e criação de valor nas empresas listadas na BM\&FBovespa. Revista Ambiente Contabil, 9(1), 364-382. DOI: 10.18405/recfin20170306

Machado, M. R., Machado, M. A. V., \& Corrar, L. J. (2009). Desempenho do Índice de Sustentabilidade Empresarial (ISE) da Bolsa de Valores de São Paulo. Revista Universo Contábil, 5(2), 24-38. DOI: http://dx.doi.org/10.4270/ruc.20095

Machado-da-Silva, C. L., \& Fernandes, B. H. R. (1999). O impacto da internacionalização nos esquemas interpretativos dos dirigentes dos dirigentes do Banco Bamerindus. Revista de Administração de Empresas, 39(1), 14-24. DOI: http://dx.doi.org/10.1590/S0034$\underline{75901999000100003}$

Maia, A. B. G. R., Vasconcelos, A. C., \& De Luca, M. M. M. (2013). Governança corporativa e internacionalização do capital social das companhias brasileiras do setor de construção e transportes. Revista Eletrônica de Negócios Internacionais, 8(2), 40-60.

Mapurunga, P. V. R., Ponte, V. M. R., \& Oliveira, M. C. (2015). Determinantes das práticas de governança corporativa: um estudo nas empresas registradas na CVM, Advances in Scientific and Applied Accounting, 8(3), 374-395. DOI: http://dx.doi.org/10.14392/asaa.2015080306

Martins, G. A., \& Theóphilo, C. N. (2009). Metodologia da investigação científica para ciências sociais aplicadas. São Paulo: Atlas.

McDonald, D., \& Puxty A. G. (1979). An inducement-contribution approach to corporate financial reporting. Accounting, Organizations \& Society, 4(1-2), 53-65. DOI: https://doi.org/10.1016/0361-3682(79)90007-2

Michelon, G., \& Parbonetti, A. (2012). The effect of corporate governance on sustainability disclosure. 
Journal of management \& governance, 16(3), 477 509.

Minussi, J. A., Damacena, C., \& Ness Jr; W. L. (2002). Um modelo de previsão de solvência utilizando regressão logística. Revista de Administração Contemporânea, 6(3), 109-128. DOI: http://dx.doi.org/10.1590/S1415-

$\underline{65552002000300007}$

McWilliams, A., \& Siegel, D. (2001). Corporate social responsibility: A theory of the firm perspective. Academy of Management Review, 28, 117-127. DOI: https://doi.org/10.5465/amr.2001.4011987

Platchek, R. B., Floriani, D. E., \& Borini, F. M. (2012). A influência do grau de internacionalização no desempenho das empresas têxteis. Revista Gestão Organizacional, 5(1), 70-81.

Ribeiro, I., Serra, F. A. R., \& Bertolini, G. R. F. (2016). Influências da experiência internacional e da diversificação de negócios no grau de internacionalização das Multinacionais Brasileiras. Revista Eletrônica de Negócios Internacionais, 11(3), 36-49. DOI: https://doi.org/10.18568/19804865.11336-48.

Ruigrok, W., \& Wagner, H. (2003). Internationalization and performance: an organizational learning perspective. Management International Review, 43(1), 63-83.

Sampieri, R. H., Collado, C. F., \& Lúcio, P. B. (2013). Metodologia de pesquisa. 3. ed. São Paulo: McGraw Hill.

Santos, J. G. C., Vasconcelos, A. C., \& De Luca, M. M. M. (2015). Internacionalização de empresas e governança corporativa: uma análise das maiores companhias abertas do Brasil. Advances in Scientific and Applied Accouting, 8(3), 300-319. DOl: 10.14392/ASAA.2015080302

Santos, P. S. A., Almeida, D. M., \& Bezerra, F. A. (2013). Grau de disclosure voluntário e nível de governança corporativa no caso das companhias brasileiras de capital aberto: um estudo de relação. Revista de Administração e Contabilidade - ReAC, 5(1), 4-21. DOI: http://dx.doi.org/10.14392/asaa.2015080302
Scholtens, B., \& Sievänen, R. (2013). Drivers of socially responsible investing: A case study of four Nordic countries. Journal of Business Ethics, 115, 605-616. DOI: 10.1007/s10551-012-1410-7

Sievänen, R., Rita, H., \& Scholten, B. (2013). The drivers of responsible investment: The case of European pension funds. Journal of Business Ethics, 117, 137-151. DOI: 10.1007/s10551-012-1514-0

Silva, M. L., Coronel, D. A., Freitas, C. A., \& Silva, R. A. (2015). O impacto da Parceria Transatlântica de Comércio e Investimento (TTIP) para as economias norte-americana e europeia. Perspectiva Econômica, 11(2), 143-154. DOI: 10.4013/pe.2015.112.05

Smaniotto, E., Paiva, E. L., \& Vieira, L. M. (2012). Estratégia de internacionalização através de upgrading funcional. Contextus: Revista Contemporânea de Economia e Gestão, 10(1), 15-29.

Soschinski, C. K., Brandt, E., \& Klann, R. C. (2019). Internacionalização e práticas de responsabilidade social corporativa em empresas brasileiras. Advances in Scientific \& Applied Accounting, 12(1), 47-64.

Souza, F. C., Murcia, F. D., \& Marcon, R. (2011). Bonding hypothesis: análise da relação entre disclosure, governança corporativa e internacionalização de companhias abertas no Brasil. Contabilidade, Gestão e Governança, 14(2), 62-81.

Sullivan, D. (1994). Measuring the degree of internationalization of a firm. Journal of International Business Studies, 34(2), 165-186.

Symeou, P. C., Zyglidopoulos, S., \& Williamson, P. (2017) Internationalization as a driver of the corporate social performance of extractive industry firms. Journal of World Business, Volume 53, Issue 1, 27-38. DOI: doi:10.1016/i.jwb.2017.07.004

Vermeulen, F., \& Barkema, H. (2002). Pace, rhythm, and scope: Process dependence in building a profitable multinational corporation. Strategic Management Journal, 23(7), 637-653. DOI: https://doi.org/10.1002/smj.243 


\section{Sobre os autores:}

Ítalo Carlos Soares do Nascimento - Universidade Federal do Ceará - UFCE, Fortaleza, CE (Brasil). Email: italocarlos25@gmail.com Orcid id: https://orcid.org/0000-0002-8151-696X

Andressa Ruth Sousa Santos - Universidade Federal do Ceará - UFCE, Fortaleza, CE (Brasil). E-mail: andressa ruth@hotmail.com Orcid id: https://orcid.org/0000-0002-3965-7929

Adriano Fleck de Paula Pessoa - Universidade Federal do Ceará - UFCE, Fortaleza, CE (Brasil). E-mail: adrianofleck@uol.com.br Orcid id: https://orcid.org/0000-0002-1846-5944

Daniel Barboza Guimarães - Universidade Federal do Ceará - UFCE, Fortaleza, CE (Brasil). E-mail: barbozadan@hotmail.com Orcid id: https://orcid.org/0000-0001-6966-7194

Sílvia Maria Dias Pedro Rebouças - Universidade Federal do Ceará - UFCE, Fortaleza, CE (Brasil). E-mail: mdpedro@gmail.com Orcid id: https://orcid.org/0000-0002-8475-9748

\section{INTERNATIONALIZATION AND BUSINESS SUSTAINABILITY IN BRAZIL}

Ítalo Carlos Soares do Nascimento, Andressa Ruth Sousa Santos, Adriano Fleck de Paula Pessoa, Daniel Barboza

Guimarães, Sílvia Maria Dias Pedro Rebouças

Universidade Federal do Ceará - UFC, Fortaleza, CE (Brasil)

\section{ARTICLE DETAILS}

Article history:

Received: 06 January 2020

Accepted: 30 June 2020

Available online August: 01 th 2020

Double Blind Review System

Scientific Editor

Ilan Avrichir

\section{Key words}

Internationalization

Corporate sustainability

Stakeholder theory

\begin{abstract}
Objective: Investigate the relationship between internationalization and corporate sustainability in companies listed in Brazil, Bolsa, Balcão (B3 S.A.).

Method: The sample gathers 95 of the 100 companies listed in B3 S.A. with the highest market value, according to data extracted from Economática ${ }^{\circledR}$. Data refer to the fiscal year 2017, being collected in November 2018, using the test of differences between means and logistic regression for quantitative analysis.

Main results: The result of the test of differences between averages revealed that only companies internationalized through physical presence in other countries showed statistically significant differences regarding corporate sustainability, which was also ratified with the application of logistic regression, noting that internationalization through social capital and revenues has not shown a relationship with corporate sustainability. Thus, the research hypothesis that there is a positive relationship between internationalization and corporate sustainability in Brazil is rejected.

Relevance/originality: The synergy between the two constructs Internationalization and Business Sustainability -, evidenced in foreign research, has demonstrated the relevance of this debate. At the national level, in turn, no empirical evidence was found that dealt with the relationship between the variables studied.

Theoretical/Methodological Contributions: The study of internationalization and corporate sustainability provides reflections on the pursuit of long-term value, thus allowing us to visualize the Stakeholder Theory, which holds that companies, in order to ensure long-term value, need to reconcile their interests. of the various stakeholders involved.
\end{abstract}




\section{INTERNACIONALIZACIÓN Y SOSTENIBILIDAD EMPRESARIAL EN BRASIL}

Ítalo Carlos Soares do Nascimento, Andressa Ruth Sousa Santos, Adriano Fleck de Paula Pessoa, Daniel Barboza Guimarães, Sílvia Maria Dias Pedro Rebouças

Universidade Federal do Ceará - UFC, Fortaleza, CE (Brasil)

\section{HISTORIA DEL ARTÍCULO}

Historia del Artículo:

Recibido: 06 de Enero de 2020

Aceptado: 30 de Junio de 2020

Disponible en línea: 01 de Agosto 2020

Double Blind Review System

Editor Científico

Ilan Avrichir

Palabras-clave:

Internationalization

Corporate sustainability

Stakeholder theory

\section{RESUMEN}

Objetivo: Investigar la relación entre internacionalización y sostenibilidad corporativa en empresas que cotizan en Brasil, Bolsa, Balcão (B3 S.A.).

Método: La muestra reúne a 95 de las 100 empresas que figuran en B3 S.A.con el valor de mercado más alto, según datos extraídos de Economática ${ }^{\circledR}$. Los datos se refieren al año fiscal 2017, que se recopiló en noviembre de 2018, utilizando la prueba de diferencias entre medias y regresión logística para el análisis cuantitativo.

Resultados principales: El resultado de la prueba de diferencias entre promedios reveló que solo las empresas internacionalizadas a través de la presencia física en otros países mostraron diferencias estadísticamente significativas con respecto a la sostenibilidad corporativa, que también se ratificó con la aplicación de regresión logística, señalando que la internacionalización a través del capital social y los ingresos no ha mostrado una relación con la sostenibilidad corporativa. Por lo tanto, la hipótesis de investigación de que existe una relación positiva entre la internacionalización y la sostenibilidad corporativa en Brasil es rechazada.

Relevancia/originalidad: la sinergia entre las dos construcciones (internacionalización y sostenibilidad empresarial), evidenciada en la investigación extranjera, ha demostrado la relevancia de este debate. A nivel nacional, a su vez, no se encontró evidencia empírica que tratara la relación entre las variables estudiadas.

Contribuciones teóricas/metodológicas: el estudio de la internacionalización y la sostenibilidad corporativa proporciona reflexiones sobre la búsqueda del valor a largo plazo, lo que nos permite visualizar la Teoría de las partes interesadas, que sostiene que las empresas, para garantizar el valor a largo plazo, necesitan conciliar sus intereses. los diversos actores involucrados

\section{Como citar este artigo:}

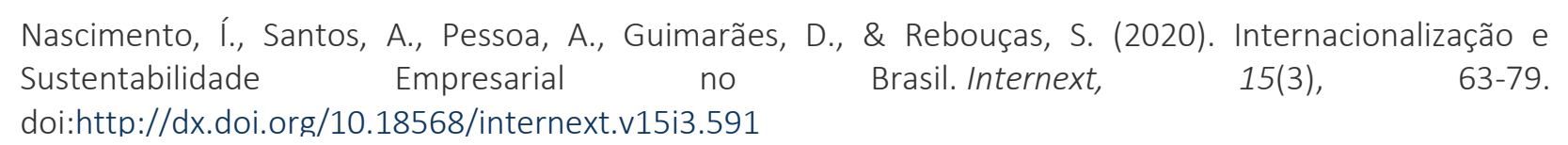

